\title{
Flexible Fiberoptic Bronchoscopy in Pediatric Practice
}

\author{
Anil Sachdev and Ritika ChHawchHaria \\ From Department of Pediatrics, Sir Ganga Ram Hospital, Rajinder Nagar, New Delhi, India. \\ Correspondence to: Dr Anil Sachdev, Department of Pediatrics, Sir Ganga Ram Hospital, Rajinder Nagar, New Delhi, India. \\ anilcriticare@gmail.com
}

Flexible fiberoptic bronchoscopy a vital diagnostic and therapeutic procedure for assessing the airway. Its logistics, clinical indications and utility need to be elucidated in pediatric context. Pediatric flexible fibreoptic bronchoscopy is useful for diagnosis of airway anomalies, bronchoalveolar lavage for diagnostic and therapeutic purposes, and interventions like foreign body removal. Newer ultrathin bronchoscopes can be used to perform this procedure in children of all ages. Pediatric flexible bronchoscopy is a valuable diagnostic and therapeutic tool in the hands of skilled personnel when used judiciously.

Keywords: Airway anomalies, Bronchoalveolar lavage, Diagnostic tool, Foreign body removal.

$\mathrm{F}$ lexible fiberoptic bronchoscopy (FFB) and bronchoalveolar lavage (BAL) have emerged as important diagnostic tools for the evaluation and treatment of children with lung and airway problems. The rigid bronchoscope is made of a metal body and can be passed through the tracheobronchial tree under general anesthesia. The major advantages of FFB as compared to rigid bronchoscopy include smaller external diameter of the new pediatric flexible scopes, the ability to change direction (flex and extend within the airway), fine illumination with fiberoptic technology and airway dynamics evaluation. Flexible bronchoscopy was introduced by Ikeda in 1968 [1]. Pioneering work by Wood and Fink popularized the use of flexible bronchoscopy in pediatrics [2]. In recent years, smaller models of flexible bronchoscope have become available thereby creating opportunities for applications that had hitherto been unthinkable. Visualization of airways by FFB has now become an integral part in the management of infants, neonates and children. Technological advances in the field of fiberoptics and cameras have opened new horizons in the field. Higher magnification and digital cameras have further enhanced the utility of FFB in neonates and children.

\section{THE EQUIPMENT}

The necessary equipment consists of a flexible bronchoscope of an appropriate size and a light source. Photographic equipment such as a still or video camera is highly desirable. Additionally, intravenous equipment, drugs used for anaesthesia and resuscitation, suction apparatus, oxygen delivery system and appropriate specimen collection containers are needed. Appropriate sized mask, endotracheal tube, laryngoscope and resuscitation bag should be easily available. It is highly desirable that advanced airway management equipments should be checked and documented to be in functional condition by the person responsible for sedation and monitoring during the procedure. The most important consideration is selecting the appropriate size of bronchoscope in children owing to their narrow airways. As the patient has to breathe around the flexible bronchoscope, the size of the bronchoscope should not be more than two-third of the diameter of the trachea (Table I). The smallest sized bronchoscope available should be used in neonates, infants and young children to reduce obstruction of the airway lumen by the bronchoscope during the procedure (which would impair

Table I Specifications of Pediatric Flexible Bronchoscopes

\begin{tabular}{|c|c|c|c|c|c|c|}
\hline Outer Diameter(mm) & Working Chamber (mm) & Suction Channel (mm) & Biopsy & Brush & ETtube & Utility \\
\hline 4.8 & 2.2 & 2.0 & Good & + & $>5.5$ & $7-8$ y or $>20 y$ \\
\hline 3.6 & 1.2 & 1.2 & Small & + & $>5$ & Standard pediatric \\
\hline 2.8 & 1.2 & 1.2 & Small & + & $>4$ & Newborn-infants-children \\
\hline 2.2 & - & No & No & - & $>3$ & Newborn and infants $<6 \mathrm{mo}$ \\
\hline 1.8 & - & No & No & - & & \\
\hline
\end{tabular}




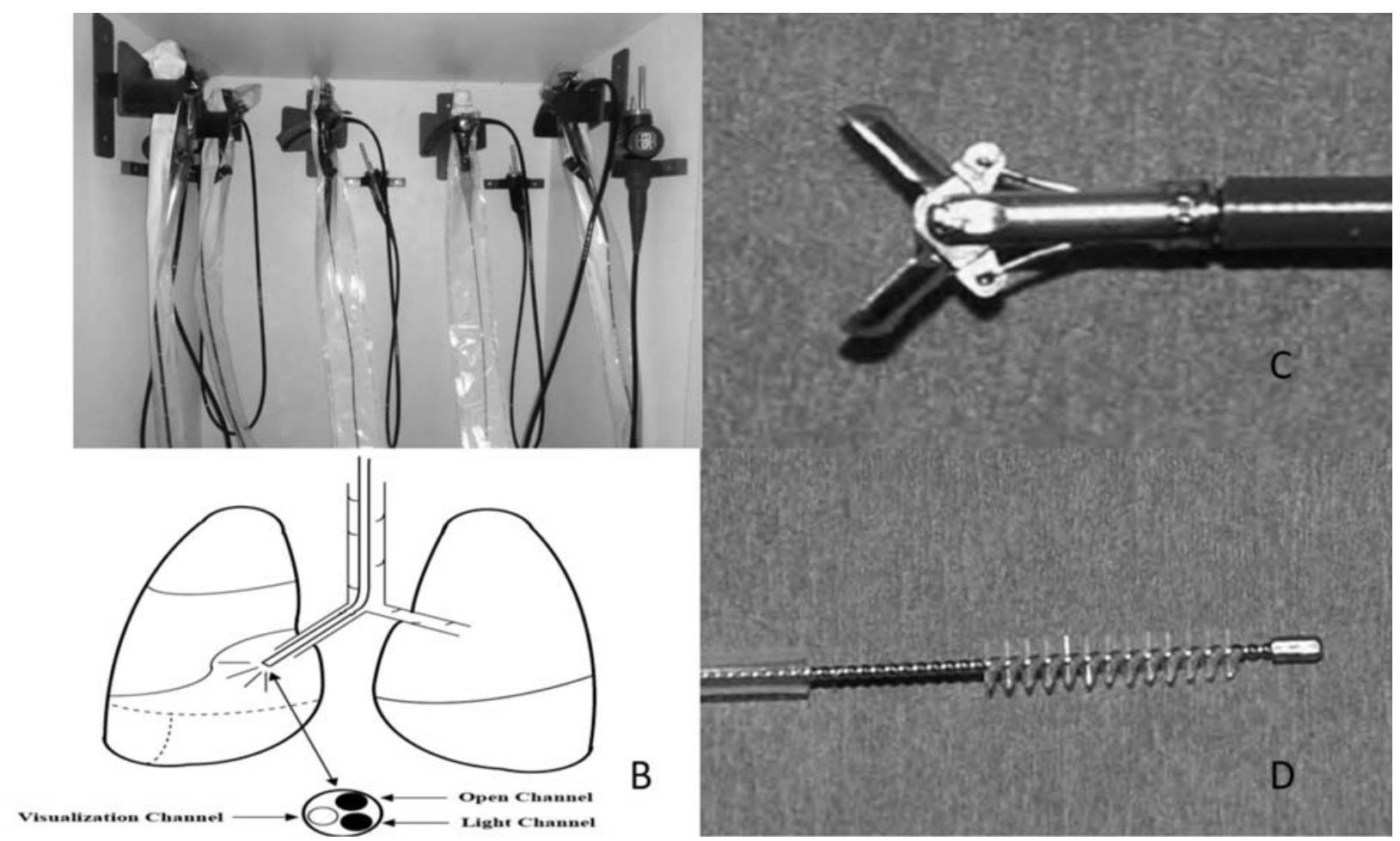

Fig. 1 A: Pediatric fiberoptic bronchoscopes; B: Placement of different channels in a flexible bronchovideoscope; C: Tip of endobronchial biopsy forceps; D: Cytology brush tip shown outside its sheath.

ventilation) and to minimize local mucosal trauma [3]. Thus, for neonates flexible bronchoscopes with outer diameter (OD) of $2.8 \mathrm{~mm}$, for infants and young children $3.6 \mathrm{~mm}$ OD scopes and for older children bronchoscope of $4.8 \mathrm{~mm}$ OD are used. Ultrathin bronchoscope (2.2 $\mathrm{mm} \mathrm{OD)}$ are used in neonates weighing less than one $\mathrm{kg}$ and during intraoperative airway assessment. Fig. 1 shows a pediatric flexible bronchovideoscope with placement of working channel and light source at the tip of scope and equipments required to obtain diagnostic material during bronchoscopy. The quality of images obtained and visualization increases with the increase in diameter (and hence the number of fiberoptic cables) of the flexible bronchoscope.

\section{SETTING}

The place where the bronchoscopy is conducted is dependent on the clinical condition of the patient, the technical and clinical abilities of the bronchoscopist and the nature of the available equipment and personnel. The procedure can be safely performed in a bronchoscopy suite, at the bedside in the intensive care unit or in the operating room. The team also consists of a technician trained in organizing the procedure, cleaning and handling the equipment and a trained nurse to prepare drugs required during procedure. A person qualified to provide sedation, monitoring and resuscitation during the procedure should support the bronchoscopist. It is recommended that one person observes and monitors the patient and another helps administer medications and assist the bronchoscopist as needed.

\section{Pre-Procedure Evaluation}

The detailed history-taking and physical examination should be performed before performing FFB. History of obstructive sleep apnea, previous anaesthetic difficulties or previous head and neck surgery should also be taken. Radiographic studies should be available for review during the bronchoscopy. The procedure is simple, well tolerated and generally requires few hours (four) of hospitalization. Prior informed consent for sedation and FFB procedure should be obtained. The child should not ingest water four hours prior to procedure or any solid food six hours prior to procedure. The various prerequisites for FFB are enlisted in Box 1 .

Established guidelines and manufacturer's recommendations for inspection, maintenance, storage, cleaning, and manual or automated reprocessing of flexible bronchoscopes, should be strictly followed. 
Box 1 PRE-REQUisites for Flexible Fiberoptic BRONCHOSCOPY

- Indication for flexible bronchoscopy

- Appropriate size bronchoscope

- Facility and skill for bronchoscopy

- Facility for monitoring during bronchoscopy

- Facility and skills for cardiopulmonary resuscitation

- Informed consent from the parents/guardians

- Microbiology and cytopathology to analyze the bronchoalveolar lavage fluid

- Facility for video-recording the bronchoscopy (preferable).

During bronchoscopy staff should wear protective clothing (gowns or plastic aprons, masks/visors, and gloves). Universal precautions are recommended for all staff that may be exposed to body secretions [3].

\section{INDICATIONS}

FFB helps obtain anatomical and dynamic information of the airways and to perform cytological and microbiological studies. The indications include symptoms or radiological anomalies that cannot be explained by non-invasive methods or to obtain samples from the lower airways [4]. The various indications for flexible bronchoscopy are highlighted in Box 2.

\section{CONTRA-INDICATIONS}

Flexible bronchoscopy is generally well tolerated. Appropriate measures to optimize the patient's condition should be taken to minimize risk. The indication for FFB should be individualized. The procedure should only be performed when the benefits outweigh the risks. The absolute contraindications that impede performing bronchoscopy are severe refractory hypoxemia, hemodynamic instability, uncorrected hemorrhagic diathesis and the lack of authorization for the procedure by the parent or guardian. The relative contraindications depend on the experience of the team and the level of critical care in the hospital. In very premature newborns and children with congenital cyanotic cardiomyopathies with an increase in bronchial collateral circulation, severe pulmonary hypertension or coagulation alterations, riskbenefit assessment must be done.

\section{SEDATION AND Monitoring}

The aim of sedation in a child is to ensure that the patient is safe, comfortable and reasonably still during the procedure while maintaining adequate oxygenation and ventilation. Bronchoscopy can be performed in sedation with benzodiazepines or narcotics or under general anaesthesia. 'Conscious sedation', where patient can follow verbal instructions and reflexes are preserved, is not recommended [4]. An anaesthesiologist or intensivist must be present to administer drugs and monitor the patient continuously during the procedure. Spontaneous respiration is preferred during diagnostic procedures, hence level of sedation should be appropriate. Diagnosing dynamic airway anomalies may be difficult in deeply sedated child with no spontaneous respiration. Use of positive pressure ventilation during the procedure helps in maintaining adequate ventilation in diagnostic procedures. Preferred drugs for local anaesthesia are $2 \%$ lidocaine jelly for the nose and $1 \%$ lidocaine spray for the pharynx and larynx. For analgesia and sedation, midazolam (0.05-0.2 mg/kg), fentanyl (1-3 mcg/kg) or ketamine (1-3 $\mathrm{mg} / \mathrm{kg})$ are used.

Box 2 IndiCATIONS OF FLEXIBLE FibEROPTIC BRONCHOSCOPY IN CHILDREN

Evaluation of airways

- Suspicion of a foreign body

- Persistent stridor

- Persistent wheezing

- Hemoptysis

- Persistent or recurring pneumonia

- Localized pulmonary hyperlucency

- Persistent or recurring atelectasis

- Problems related with the artificial airways

- Miscellaneous (large burns, phonatory anomalies)

Obtaining cultures (bronchoalveolar lavage, bronchial biopsy)

- Pneumonia in immunosuppressed patients

- Chronic interstitial pneumonia

- Pneumonitis due to hypersensitivity

- Pulmonary hemosiderosis

- Eosinophilic pneumonia

- Other (sarcoidosis, alveolar proteinosis, histiocytosis)

- Endoluminal obstructive pathology

- Aspiration lung syndromes

Therapeutic indications

- Difficult or selective intubations

- Aspiration of endobronchial secretions

- Instillation of medication

- Management of the foreign body combined with rigid bronchoscope 
Monitoring of the patient includes continuous evaluation of heart rate, respiratory rate, color, head position and assessment of gas exchange by continuous pulse oximetry. Continuous ECG and invasive monitoring are desirable in sick critical patients like children with cardiac diseases. A sedated patient should not be left alone or unobserved. The pediatric patient must be adequately monitored until awake. Supplemental oxygen should be maintained after the procedure until patient has recovered from sedation and adequate oxygenation on room air is documented. When the procedure is performed on an outpatient, the child should be tolerating oral intake prior to discharge.

\section{Route AND Procedure}

Flexible bronchoscopy is usually performed trans-nasally and can be performed orally or via artificial airway. Oxygen mask or nasal prongs can be used simultaneously while using the nasal route or the oral route. Nasal route can be employed while the patient is on high flow nasal cannula ventilation. Special noninvasive ventilation facemasks (interfaces) are available that can be used for FFB while patient continues to receive positive pressure ventilation. Oral route is used when nasal route is not feasible as in choanal atresia, nasal bleeding or trauma. Laryngeal mask airway can be used for ventilation through oral route. Endotracheal tubes and tracheostomy tubes of minimum size of $4 \mathrm{~mm}$ are used in critically ill patients with an appropriate size bronchoscope [5].

The operator stands or sits at the head of the patient with the gurney in low position in order to avoid the equipment from getting curved. Forced curves in the scope can damage its fibers and make it difficult to handle. An appropriate sized bronchoscope is chosen according to the child's age. The anatomy and the functionality of the pharyngeal and laryngeal structures (sublingual glands, tonsils, arytenoids, epiglottis and vocal cords) are studied if the access is nasal. Further progression through the larynx is done by centering the end of the bronchoscope in the angle of the anterior corner of the vocal cords, introducing it by means of posterior flexion when the patient inhales. In order to make the passage easier and to prevent the appearance of laryngeal spasm, a local dose of $1 \%$ lidocaine can be sprayed through the working channel. After reaching the subglottic space, lidocaine spray and proceed method is used to suppress cough while negotiating trachea and bronchi. The bronchoscope is further advanced until wedged in a desired sub-segmental bronchus at the desired location.

Bronchoalveolar lavage: BAL is usually carried out in the most-affected area (identified radiologically and/or endoscopically). The right lower lobe offers better fluid recovery and is the preferred site for BAL in diffuse lung disease. In infants it is often easier to perform BAL in the right lower lobe. If BAL and trans-bronchial biopsies are planned in the same patient, BAL should be performed first. BAL is carried out using normal sterile saline previously warmed to body temperature. Limited information exists on the amount of fluid and the number of aliquots that should be used in order to obtain samples representative for the alveolar compartment in children of different ages and sizes. The amount of fluid instilled can be determined using body weight as $3 \mathrm{~mL} / \mathrm{kg}$ of normal saline divided into three equal fractions for children weighing $<20 \mathrm{~kg}$ and $3 \mathrm{~mL} / \mathrm{kg}$ in $20 \mathrm{~mL}$ portions in children weighing $>20 \mathrm{~kg}$ [5]. The aliquots are instilled using a syringe via the suction channel of the FFB and then gentle suction $(50-80 \mathrm{mmHg})$ is applied to collect the lavage specimen in the collection trap [6]. The minimum amount of BAL fluid necessary to perform the typical battery of laboratory tests depends on the clinical scenario and endoscopic findings. For adults it is recommended that the minimal total volume retrieved is $>30 \%$ of the instilled volume [3]. The BAL fluid can be sent for investigations and can aid in diagnoses (Box 3). Centers for Disease Control/National Health Safety Network criteria for ventilator-associated pneumonia (VAP) with common bacterial organisms specifies diagnostic threshold values as $>10^{4}$ colony-forming units (cfu) $/ \mathrm{mL}$ from BAL and $>10^{3} \mathrm{cfu} / \mathrm{mL}$ from protected specimen brushing [7]. BAL results have been used as a reference test for the diagnosis of VAP [8]

\section{Diagnostic UTILITY}

\section{Flexible Bronchoscopy}

Flexible bronchoscopy allows direct visualization of the upper and lower airway, enabling detection of anatomical defects that may not be visible on radiologic imaging. Presence of endobronchial infections, granulations, tumors can be confirmed by FFB. Also, it allows dynamic changes in the airway, and hence diagnosis of dynamic airway obstructions like airway malacias and excessive dynamic airway compression (EDAC) is possible only by FFB which may be missed on virtual bronchoscopy obtained with computerized tomography of chest. Flexible bronchoscopy along with dynamic CT (not very readily available) is used to diagnose EDAC and differentiate it from tracheobronchomalacia in adults [9]. Apart from anatomical delineation, FFB also helps in collection of bronchoalveolar lavage and biopsies that can be subjected to microbiological, pathological examination. Bronchoscopy can be indicated in children with unusual presentations of chronic cough or wheeze, 
Box 3 POSSIBLE INVESTIGATIONS IN BRONCHOALVEOLAR LAVAGE FLUID

\section{Cell count and differential}

- Alveolar macrophages (Normal $>80 \%$ )

- Neutrophils (Normal <3\%)

- Eosinophilia (Normal $<1-2 \%$ )

- Lymphocytosis (Normal $<15 \%$ )

- Erythrocytes

Microbiology

- Cultures

- Stains and Immunohistochemistry : Gram stain; $\mathrm{KOH}$ stain; Periodic acid-Schiff (PAS); Direct fluorescent stain (DF) or Ziehl-Neelson (ZN stain); Modified acid fast stain (Kinyoun); Giemsa stain; Inclusion bodies

- Polymerase chain reaction (PCR): Mycobacteria tuberculosis and numerous pathogens.

- Quantitative or semiquantitative cultures: particularly for ventilator associated pneumonia.

- Diagnostic of infection if organism identified: Pneumocystis carinii, Strongyloides etc.

Cytology

- Foamy macrophages

- Malignancies

- Sulfur granules

- Hemosiderin-laden macrophages

- Langerhans cells: $>5 \%$ suggestive of Pulmonary Langerhans cell histiocytosis;

- Fat and Lipid stain (e.g. Sudan III): Lipoid pneumonia (aspiration)

and cystic fibrosis [10]. Few bronchoscopic diagnoses are shown in Web Fig. 2. Bar-Zohar, et al. [11] could detect airway pathologies in $69 \%$ of PICU patients who underwent bronchoscopy for suspected airway malformations.

\section{Endobronchial Ultrasound (EBUS)}

Ultrasound probes (balloon-tipped, miniaturized) are passed through the working channel of flexible bronchoscope to visualize the tracheobronchial wall and immediate surrounding structures. It is useful in lymphoma and mediastinal tumors where the extent of lymphnodes can be determined, avoiding a CT scan. The probe is pressed against the bronchial wall and balloon is inflated. A biopsy can be obtained from the adjacent lymph node for diagnosis of tuberculosis, sarcoidosis, malignancy etc. [12].

\section{Therapeutic Procedures}

Aspiration of secretions: FFB can be useful for resolving atelectasis due to the retention of secretions or mucus plugging. Repeated large volume BAL is the recommended treatment in pulmonary alveolar proteinosis and in acquired conditions like lipoid pneumonia [13,14].

Difficult and selective intubations: Bronchoscopy can act as a guideline for intubation in cases of craniofacial anomalies and multiple malformations syndromes [15]. It has also been used for selective bronchial intubation and intubation in special conditions like suspected or proven cervical spine injuries [16].

Removal of foreign body: The removal of foreign body using FFB is a complicated procedure in children. Some publications endorse the good performance of FFB for foreign body removal [17]. Though FFB is the procedure of choice for diagnosis of foreign body, rigid bronchoscopy remains the gold standard for its retrieval in children and in adults [18]. In children, rigid bronchoscopy is preferred as it offers the advantages of general anesthesia, assisted ventilation, larger instruments and a greater variety of accessories. The ideal procedure would be to initiate with FFB, which allows for greater reach in the exploration and the identification of the foreign body, extraction with rigid bronchoscope, and if required, a final revision with FFB to rule out a residual foreign body.

Transbronchial biopsy: Transbronchial biopsy (TBB) involves obtaining a biopsy sample of lymph nodes surrounding the carina or of the pulmonary parenchyma for microscopic analysis (Fig. 1). The samples from lymph nodes helpin diagnosing conditions like extra-pulmonary tuberculosis and lymphoma. The samples from pulmonary parenchyma are preferred for diagnosis of rejection in children with lung transplant. The size of the TBB sample obtained determines the yield of TBB. Multiple small sized lymph node samples are better while fewer larger samples may be required to accurately diagnose cancer and ILDs [19].

Bronchial washing: Bronchial washing can be used to study the bronchial mucosa or to culture bronchial secretions (Fig. 1). It is a useful technique for the diagnosis of ciliary dyskinesia, tuberculosis and pneumonia in patients with mechanical ventilation and lung infections in immunosuppressed patients.

Balloon dilatation: Balloon dilatation of stenosed or narrow airways can be done by rigid bronchoscopy and FFB. Ideal cases for balloon dilatation are web-like stenosis, benign strictures, complication of endotracheal intubation or granulomatous disease. It is a minimally invasive, safe and rapid procedure [20]. 


\section{Pitfalls}

There are situations where radiological imaging may take precedence over FFB. Especially when there is a lack of expertise or infrastructure required to ensure safety of the procedure. In cases of persistent pneumonia with poor response to antibiotic treatment, BAL can be obtained specifically from affected sites that are identified on a CT scan., The diagnostic yield of FFB alone is low in peripheral lung nodules in malignancies or infections (fungal or tubercular) [21]. In congenital parenchymal lung malfor-mations, CECT is the investigation of choice for diagnosis. FFB aids to identify associated airway anomalies [22]. This helps in planning the type of surgery and need for airway reconstruction, postoperative management [4].

\section{Cleaning and Disinfection}

Cleaning and disassembling of the flexible bronchoscope is a delicate procedure because of the complex valves and channels. Bronchoscopes need to be cleaned and disinfected thoroughly after every use in a dedicated room to prevent cross-infection amongst patients. The bronchoscopes must be checked for leaks to prevent leakage of fluid into the optic system. After disassembling the parts, thorough cleaning with tap water or detergent water is necessary. Preferred agent for disinfection of cleaned bronchoscope is $2 \%$ glutaraldehyde. Bronchoscopes should be immersed in this solution for $20 \mathrm{~min}$ to ensure killing of all pathogens. Disinfection should be followed by rinsing with deionized water to remove all the disinfectant from the bronchoscope. Gas sterilization with ethylene oxide at temperature $<55^{\circ} \mathrm{C}$ is safe but not always practical. Conventional heat sterilization methods can destroy the bronchoscope. Removable, heat-stable parts like suction valve etc can be steam autoclaved. Damaged bronchoscopes should be gas sterilized as they cannot be immersed in disinfectant [23].

\section{Complications}

Pediatric bronchoscopy is a generally well-tolerated procedure. Bronchoscopy-related complications can be broadly classified into $(i)$ complications due to anesthesia (which account for $50 \%$ of the complications); and (ii) complications due to bronchoscope in the airway. Hypoxemia may occur during the procedure requiring initiation of supplemental oxygen. Increased airway resistance, excessive sedation, and disturbance of ventilation-perfusion relationship can cause hypoxemia. In patients who are hemodynamically unstable and on mechanical ventilation, FFB can negatively affect lung compliance and airway resistance. Inadequate use of topical anesthesia may result in adverse reactions such as laryngospasrn, bradycardia, or other vagal nerve mediated phenomena. Inadequate sedation may lead to patient discomfort. On the other hand, excessive use of sedation may result in depression of respiration. Postbronchoscopy fever may occur, especially following bronchoalveolar lavage. Mechanical complications of bronchoscopy may include epistaxis, pneumothorax, and hemoptysis. Trans-bronchial biopsy is the most common cause of bronchoscopy related hemoptysis. Rarely, infections complicating FFB are seen due to procedural and disinfection lapses [23].

\section{CONCLUSION}

FFB in neonates, infants and children has many diagnostic and therapeutic benefits. Bronchoalveolar lavage and lung tissue obtained with FFB can aid in the diagnosis of many pulmonary diseases. Dynamic airway conditions like laryngomalacia, tracheobronchomalacia can be diagnosed and quantified accurately with FFB under sedation. Proper pre-procedure preparation and monitoring during and after FFB should be followed to minimize complications. All neonatologists and pediatricians should be aware of the indications and utilities of FFB in pediatric patients.

Contributors: Both authors contributed to review of literature, manuscript writing and its approval, and are accountable for all aspects related to the review.

Funding: None; Competing interest: None stated.

\section{REFERENCES}

1. Ikeda S. Recording of the endoscopic picture. J Jap Med Instr.1967;37:291.

2. Wood RE, Sherman JM. Pediatric flexible bronchoscopy. Ann Otol Rhinol Laryngol. 1980;89:414-6.

3. Faro A, Wood RE, Schechter MS, Leong AB, Wittkugerl E, Abode $\mathrm{K}$, et al. Official American Thoracic Society technical standards: flexible airway endoscopy in children. Am J Respir Crit Care Med. 2015;191:1066-80.

4. Wood RE, Boesch RP. Bronchoscopy and bronchoalveolar lavage in pediatric patients. In: Wilmott RW, Boat TF, Bush A, Chernick V, Deterding RR, Ratjen F, editors. Kendig and Chernick's disorders of the respiratory tract in children. 8th ed. Philadelphia, PA: Elsevier Saunders; 2012. p.131-44.

5. Grigg J, van den Borre C, Malfroot A, Pierard D, Wang D, Dab I. Bilateral fiberoptic bronchoalveolar lavage in acute unilateral lobar pneumonia. J Pediatr. 1993;122:606-8.

6. De Blic. Flexible Bronchoscopy. In: Eber E, Midulla F, editors. ERS Handbook of Pediatric Respiratory Medicine. 1st ed. UK: Charlesworth Press; 2013. p.132-9.

7. Stokes DC, Shenep JL, Parham D, Bozeman PM, MarienchekW, Mackert PW. Role of flexible bronchoscopy in the diagnosis of pulmonary infiltrates in pediatric patients with cancer. J Pediatr. 1989;115:561-7.

8. Sachdev A, Chugh K, Sethi M, Gupta D, Wattal C, Menon $\mathrm{G}$. Diagnosis of ventilator-associated pneumonia in children in resource-limited setting: A comparative study of bronchoscopic and nonbronchoscopic methods. Pediatr Crit Care Med. 2010;11:258-66. 
9. Murgu S, Stoy S. Excessive dynamic airway collapse: A standalone cause of exertional dyspnea? Ann Am Thorac Soc. 2016;13:1437-9.

10. Nicolai $\mathrm{T}$. The role of rigid and flexible bronchoscopy in children. Paediatr Respir Rev. 2011;12:190-5.

11. Bar-Zohar D, Sivan Y. The yield of flexible fiberoptic bronchoscopy in pediatric intensive care patients. Chest. 2004;126:1353-9.

12. Bolliger CT, Mathur PN, Beamis JF, Becker HD, Cavaliere $\mathrm{S}$, Colt $\mathrm{H}$, et al. ERS/ATS Statement on Interventional Pulmonology. European Respiratory Society/American Thoracic Society. Eur Respir J. 2002;19:356-73.

13. Garg G, Sachdev A, Gupta D. Pulmonary alveolar proteinosis. Indian Pediatr. 2009;46:521-3.

14. Sachdev A, Anand P, Gupta D. Lipoid pneumonia- An unusual cause of acute respiratory distress syndrome. Indian Pediatr. 2015;52:63-4.

15. Finer NN. Flexible fiberoptic bronchoscopy. In: Spitzer $\mathrm{AR}$, editors. Intensive care of the fetus and neonate. Mosby, St.Louis, 1996. p. 531-7.

16. Pandharikar N, Sachdev A, Gupta N, Gupta S, Gupta D. Chest trauma: A case for single lung ventilation. Indian $\mathrm{J}$ Crit Care Med. 2016;20:248-50.

17. Kapoor R, Chandra T, Mendpara H, Gupta R, Garg S.
Flexible bronchoscopic removal of foreign bodies from airway of children: Single center experience over 12 years. Indian Pediatr.2019; 56:560-2.

18. Salih AM, Alfaki M, Alam-Elhuda DM. Airway foreign bodies: A critical review for a common pediatric emergency. World J Emerg Med. 2016;7:5-12.

19. Sehgal IS, Bal A, Dhooria S, Gupta N, Ram B, Aggarwal AN, et.al. Predictors of successful yield of transbronchial lung biopsy in patients with sarcoidosis. J Bronchology Interv Pulmonol. 2018:25,31-6.

20. Ernst A, Silvestri GA, Johnstone D, American College of Chest Physicians. Interventional Pulmonary Procedures: Guidelines from the American College of Chest Physicians. Chest. 2003;123:1693.

21. De Roza MA, Quah KH, Tay CK, Toh W, Li H, Kalyanasundaram $\mathrm{G}$, et al. Diagnosis of periphera lung lesions via conventional flexible bronchoscopy with multiplanar CT planning. Pulm Med. 2016;2016:5048961.

22. Sachdeva A, Chhawchharia R, Gupta D, Gupta N. Flexible fiberopic bronchoscopy directed interventions in neonatal intensive care unit. Indian Pediatr.2019;56:563-6.

23. Terkawi RS, Altirkawi KA, Terkawi AS, Mukhtar G, AlShamrani A. Flexible bronchoscopy in children: Utility and complications. Int J Pediatr Adolesc Med. 2016;3:18-27. 